\title{
Impact of Friction and Gait Parameters on the Optimization of Series Elastic Actuators for Gait Assistance
}

\author{
Florian Stuhlenmiller ${ }^{\mathrm{a}, *}$, Daniel Clos ${ }^{\mathrm{b}}$, Stephan Rinderknecht ${ }^{\mathrm{a}}$, Philipp \\ Beckerle $^{\mathrm{a}}$, Josep M. Font-Llagunes ${ }^{\mathrm{b}}$ \\ ${ }^{a}$ Institute for Mechatronic Systems in Mechanical Engineering, Technische Universität \\ Darmstadt, Darmstadt, Germany \\ ${ }^{b}$ Department of Mechanical Engineering and Research Centre for Biomedical Engineering, \\ Universitat Politécnica de Catalunya, Barcelona, Spain
}

\begin{abstract}
Elastic actuators feature increased energy efficiency and improved human-robot interaction compared to directly driven concepts for active orthoses and prostheses. Structure and parameters of the elastic actuation system are often designed via a model-based minimization of energy consumption based on gait data gained from healthy individuals. However, natural motion exhibits variability among individuals and may not consider requirements of persons using assistive devices. A parametric study is performed examining the impact of varying gait characteristics on the energy consumption and constraints of an optimized (clutchable) series elastic actuator of the knee joint. Furthermore, friction parameters are varied to analyze the impact on actuator constraints. Results of the parametric study indicate increased energy consumption for a slower cadence compared to the healthy gait data for both systems. The clutchable series elastic actuator is less impacted by constraints than the series elastic actuator. The utilized models are evaluated experimentally at a test bench, indicating good accordance to the measured energy consumption. The results highlight the interrelation of friction and gait parameters with energy consumption and actuator constraints and indicate that the optimization procedure for the actuator design requires detailed models of component efficiency as well as subject-specific gait characteristics.
\end{abstract}

Keywords: Series elasticity, Compliant actuators, Energy efficiency, Design optimization

\footnotetext{
* Corresponding author

Email address: stuhlenmiller@ims.tu-darmstadt.de (Florian Stuhlenmiller)
} 


\section{Introduction}

Spinal cord injuries damage or break the connections between extremity and brain, thus causing sensory and motor deficiencies. Hence, after suffering such injury, persons are partly or completely paralyzed depending on the severity

5 and location of their lesion. The quality of life of people who experienced spinal cord injury, is reduced distinctly [1]. The limited mobility can lead to dependence on caregivers and even simple tasks can become challenging. In the $\mathrm{WHO}$ global disability action plan 2014-2021, the world health organization proposes to strengthen assistive technologies to allow people with disabilities to live in dignity and achieve their full potential [2]. Affected people rate the recovery of mobility as a highly desired goal [3].

Active orthoses and prostheses are examples of assistive technologies as they complement human motor functions [4, 5, introduce and dissipate energy [6], and thereby assist the ambulation of an individual with a lower limb pathol-

15 ogy [7]. Such wearable robots show high potential to provide therapeutic benefits, however, several challenges have to be addressed before their widespread applicability in society [4, 8]. Besides the high cost of available devices [4, issues regarding sensors to detect user intention and state of the gait cycle, as well as materials for lightweight and cost-efficient design remain open [8]. Finally, high power requirements result in heavy actuators and batteries, limiting the overall performance [8]. The analysis of these constraints is the key aspect of the present study, which analyzes the parametric sensitivity of energy consumption in different elastic actuators and the impact of gait and actuator parameters. Current approaches to cope with the challenging actuator requirements and its 25 design consequences, suggest using elastic actuators with parallel springs to reduce the required actuator torque [9, 10, allowing to select a lighter drive unit. For systems with harmonic trajectories, like the human gait, series elastic actuators can improve the efficiency [11, as energy can be stored and released in the springs, while potentially reducing the actuator's velocity [12. In addition,

so elastic actuators display improved backdriveability of the load [13] and safety in robot-human interaction [14]. The appropriate selection of actuation concept and design parameters is required to actually benefit from elastic actuation. Therefore, optimizations of series and parallel stiffness, motor and transmission system are performed, often focusing on minimizing peak power or energy

35 consumption [15, 16. Another approach for the selection of spring stiffness is to passively mimic the torque-angle characteristic of human joints to achieve a natural motion as well as a comfortable human-robot interaction [17, 18. Both approaches are mostly based on average motion and torque of movement data, determined from gait analyses with healthy participants, to enable the user to

40 perform these daily living activities. However, natural motion exhibits a distinct variability between healthy individuals [19, 20, 21, while motion data of people using assistive devices are usually not available. Louie et al. [22] for example report an average walking speed of $0.26 \mathrm{~m} \mathrm{~s}^{-1}$ with a standard deviation of $0.15 \mathrm{~m} \mathrm{~s}^{-1}$ achieved by exoskeletons in different studies. Thus, the walking 45 velocity is distinctly slower than the self-selected velocity of healthy participants 
for slow walking of $1 \mathrm{~m} \mathrm{~s}^{-1}[23$. Furthermore, the high standard deviation indicates variability of the gait depending on the level of injury and practicing time [22]. Hence, an actuator design optimized based on healthy gait data may not be the optimal solution.

so In addition, recent investigations emphasize the importance of component efficiencies to correctly estimate peak power and energy consumption of elastic actuators [24, 25, 26]. However, components are not known in detail when an actuation concept is selected, thus, it is not possible to consider non-linear losses in the optimization procedures. Hence, this work examines the impact of

55 varying gait parameters and the interrelation with component efficiency on two optimized elastic actuation concepts to guide optimal elastic actuator design. Therefore, Section 2 presents the model-based optimization process while considering efficiency models of deployed components of two elastic knee joint actuators for an active knee orthosis: a series elastic actuator focused on the minimization of energy consumption, and a clutchable series elastic actuator mimicking the quasi-stiffness of the knee during the stance phase. A parametric study is performed in Section 3 to examine the impact of friction and gait parameters on the optimized elastic actuators. Section 4 presents an experimental examination of the performance of both actuation concepts to evaluate

65 the models utilized throughout this work. Finally, a discussion and summary conclude the paper.

\section{Selection of Elastic Actuation Concept for the Knee Joint}

To define the desired knee dynamics of the active orthosis, gait data of healthy individuals walking at very low speed is considered [23. Figure 1 70 presents the corresponding knee flexion angle $\theta_{k}$, knee extension torque $\tau_{k}$, the mechanical knee power $P_{k}$, and the knee torque-angle characteristic. As the knee extension torque is given in relation to the mass of the user, the calculated knee power is given in $\mathrm{W} \mathrm{kg}^{-1}$ as well. Angle and torque data are approximated by a Fourier Series to obtain smooth and continuous data, from which velocity and

75 acceleration curves were obtained by numerical derivation. The dotted black line indicates $50 \%$ of the of the stride time. As seen in the bottom right, the torque-angle relationship exhibits an almost linear behavior during the stance phase, which can be described as quasi-stiffness [27, while the characteristic in the swing phase is nonlinear and dissipative. This dissipative behavior of

so the knee joint during walking is furthermore observed in the negative values of the mechanical power during the second half of the cycle. The desired knee dynamics can be generated by different actuation concepts. An optimal concept can be selected by comparison of different systems with optimized parameters. However, at the conceptual stage of actuator selection, detailed loss models are 85 usually not available. Thus, a white-box optimization and therefore the concept selection depends on models describing the general behavior of components. For example, losses in a gear unit may be described by friction models, however, data given by manufacturers usually only provide a constant efficiency. In order to evaluate the potential impact on actuator selection, the optimization 
9o and comparison of a series elastic actuator (SEA) and clutchable series elastic actuator (CSEA) will be performed. Thereby, the calculation of peak power and energy consumption relies on available data for off-the-shelf components where possible.

\subsection{Elastic Actuator Models and Power Calculation}

A SEA powering the knee can be modeled according to Fig. 2. The actuator generates a torque $\tau_{a}$ and the interaction with the knee extension torque results in a deflection of the spring with stiffness $k_{s}$. Actuator angle and inertia are described by $\theta_{a}$ and $I_{a}$, respectively.

The dynamic equations of a SEA powering a knee joint are given by:

$$
\left[\begin{array}{cc}
I_{a} & 0 \\
0 & 0
\end{array}\right]\left[\begin{array}{l}
\ddot{\theta}_{a} \\
\ddot{\theta}_{k}
\end{array}\right]+\left[\begin{array}{cc}
k_{s} & -k_{s} \\
-k_{s} & k_{s}
\end{array}\right]\left[\begin{array}{c}
\theta_{a} \\
\theta_{k}
\end{array}\right]=\left[\begin{array}{c}
\tau_{a} \\
-\tau_{k}
\end{array}\right]
$$

As $\tau_{k}$ and $\theta_{k}$ are given by the gait data, the actuator motion is determined by the lower part of (1):

$$
\theta_{a}=\frac{\tau_{k}}{k_{s}}+\theta_{k}
$$

Inserting $\theta_{a}$ as well as the second derivative $\ddot{\theta}_{a}$ of 2 into the upper part of (1) yields the actuator torque

$$
\tau_{a}=I_{a}\left(\frac{\ddot{\tau}_{k}}{k_{s}}+\ddot{\theta}_{k}\right)+\tau_{k}
$$
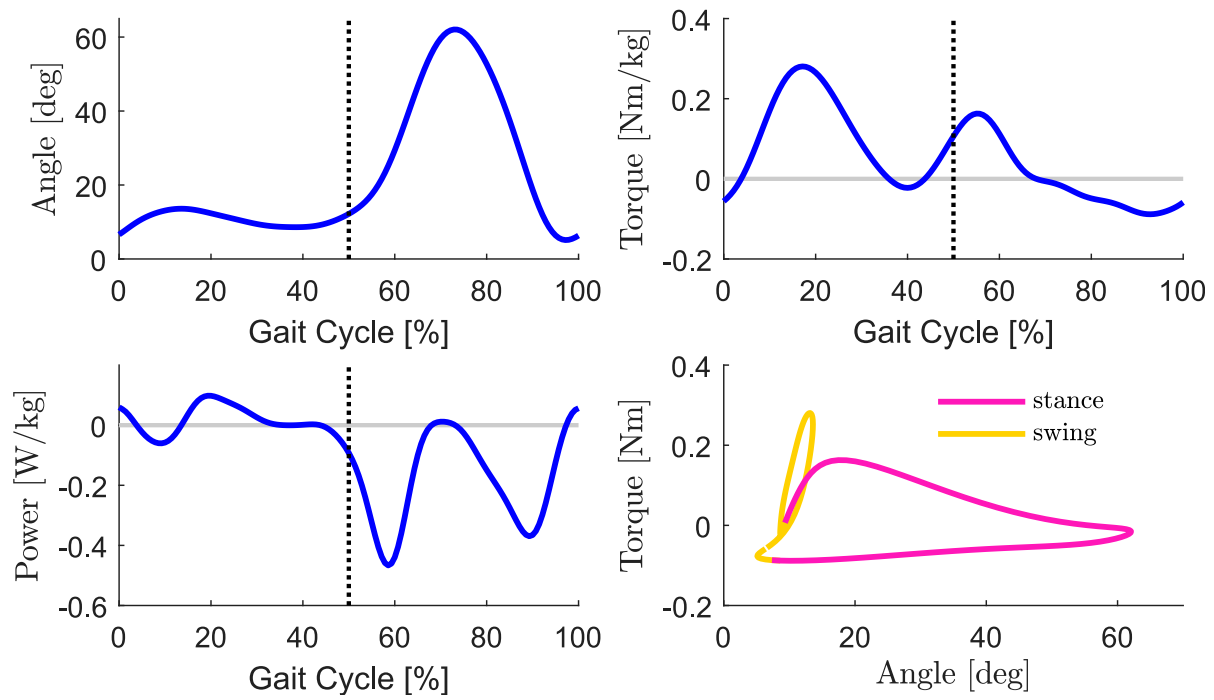

Figure 1: Gait data of the knee joint for very slow walking 23], dotted black line indicates $50 \%$ of the of the stride time. The torque-angle characteristic in the bottom right characterizes the stiffness of the knee joint. 


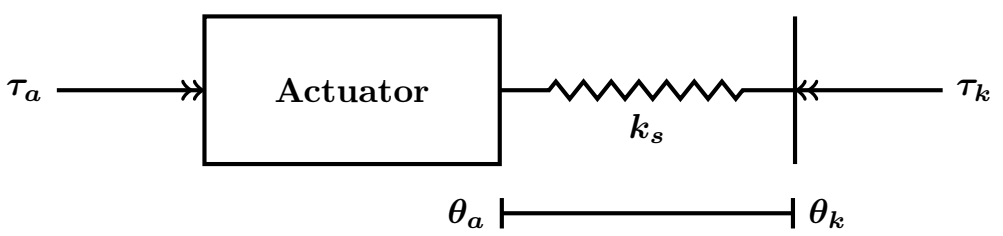

Figure 2: Model of a SEA powering the knee

95 the model can be detailed by including mechanical and electrical losses in the drive unit. The applied modeling approach follows the procedure presented in 28. To cover mechanical transmission losses, a constant gear unit efficiency of $\eta_{g u}=0.7$ is assumed. Thus, depending on the flow of power, the knee torque $\tau_{a}$ when driving the load or decreases it when being driven by the load, respectively. Assuming that the inertia of the gear unit is small compared to the reduced motor inertia, the actuator inertia is simplified to $I_{a}=i_{G}^{2} I_{m}$, with the gear ratio $i_{G}$ and the motor inertia $I_{m}$. Consequently, the torque required Thus, with $\tau_{a}=i_{G} \tau_{m}$, the required motor torque can be calculated by:

$$
\tau_{m}= \begin{cases}I_{m} i_{G}\left(\begin{array}{ll}
\ddot{\tau}_{k} \\
k_{s}
\end{array} \ddot{\theta}_{k}\right)+\frac{1}{\eta_{g u} i_{G}} \tau_{k} & \text { positive power/consumption } \\
I_{m} i_{G}\left(\frac{\ddot{\tau}_{k}}{k_{s}}+\ddot{\theta}_{k}\right)+\frac{\eta_{g u}}{i_{G}} \tau_{k} & \text { negative power/recuperation }\end{cases}
$$

The efficiency of the electric motor can be considered using models that include mechanical and resistive losses. Thereby, the electrical current is given by:

$$
I=\frac{\tau_{m}+\nu_{m} \dot{\theta}_{a} i_{G}}{k_{m}}
$$

where the torque constant is $k_{m}$, and $\nu_{m}$ is the viscous damping coefficient of the motor. The latter value is usually not given in datasheets and is therefore estimated from the no-load current $I_{n l}$ and the no-load speed $\omega_{n l}$ according to [28]:

$$
\nu_{m}=\frac{k_{m} I_{n l}}{\omega_{n l}}
$$

The knowledge of the current allows the determination of the voltage via:

$$
U=L \dot{I}+R I+k_{b} \dot{\theta}_{a} i_{G} .
$$

where the terminal resistance is denoted $R$, the terminal inductance $L$, and the speed constant of the motor $k_{b}$. Due to the small value of the terminal inductance, the component $L \dot{I}$ in $(7)$ is neglected, which is convenient if the torque does not contain high frequency components with high amplitudes, which 
applies to normal walking motion at the knee joint level 29 .

The overall motor efficiency is determined by the ratio between mechanical and electrical power:

$$
\eta_{m}=\frac{P_{m}}{P_{m, e l}}
$$

In addition, the electrical losses are also affected by the efficiency of the motor driver $\eta_{m d}$. When the motor is driven by the load, the mechanical actuator power is negative and the electrical motor is utilized as a generator. In this operation mode, the efficiency of component reduces the recuperation capabilities and thus increases power consumption $P_{s}$. Therefore, the power requirements are calculated mode-specifically:

$$
P_{s}=\left\{\begin{array}{cl}
\frac{1}{\eta_{m d}} P_{m, e l} & \text { positive power } / \text { consumption } \\
\eta_{m d} P_{m, e l} & \text { negative power } / \text { recuperation }
\end{array}\right.
$$

\subsection{Constrained Optimization of a Series Elastic Actuator}

In the following, a minimization of the required energy per gait cycle is performed by optimizing parameters of the actuation system while requiring that the actuator reproduces the angle and torque of the knee presented in Fig. 1. While the scope of this paper is not the optimization of an electric motor itself, motor parameters are required for the efficiency models. Thus, the $70 \mathrm{~W}$ brushless direct current motor EC45 flat (Maxon Motor AG, Sachseln, Switzerland) is preselected due to a high power-to-weight ratio. The motor driver EPOS 24/5 (Maxon Motor AG, Sachseln, Switzerland) is considered as a servocontroller. The optimization is implemented considering the required energy per gait cycle $E_{g c}$ as the objective function:

$$
\min \left(\int_{0}^{t_{g c}} P_{s}\left(k_{s}, i_{G}\right) \mathrm{d} t\right)
$$

with the time of one gait cycle $t_{g c}$ as well as series stiffness and gear ratio as input parameters. In addition to the efficiency, constraints of components may limit the system performance and have to be considered in the optimization. The stiffness, and the gear ratio are required to be greater than zero to respect the flow of power. The maximum torque of the motor is limited by the maximum current of the motor driver $I_{m d, \max }$ while the maximum allowable continuous current $I_{m, \text { con }}$ prevents overheating of the motor. The maximum speed of the

motor is limited by the bearings and given by $\dot{\theta}_{m, \max }$. In addition, the motor voltage is constrained to consider the maximum voltage of a power supply $U_{\max }$. The parameters and constraints of the preselected motor and motor driver are given in Table 1

Further limitations, e.g., maximum speed or peak torque of the gear unit, may impose additional constraints, but are not considered before selecting specific components. The impact of extended constraints on the optimal solution could 
be investigated in a second iteration of the actuator design process. For the given constraints, the solution of the optimization has to satisfy:

$$
\left[\begin{array}{c}
-k_{s} \\
-i_{G} \\
\max (|I|) \\
\operatorname{mean}\left(I_{m}\right) \\
\max \left(\left|\dot{\theta}_{m}\right|\right) \\
\max (|U|)
\end{array}\right]<\left[\begin{array}{c}
0 \\
0 \\
I_{m d, \text { max }} \\
I_{m, \text { con }} \\
\dot{\theta}_{m, \text { max }} \\
U_{m}
\end{array}\right]
$$

The constrained optimization problem is implemented in Matlab (MathWorks, Natick, Massachusetts, USA) via the gradient-based fmincon function. For a gait cycle with $t_{g c}=1.4 \mathrm{~s}$ and a subject's mass of $m_{h}=75 \mathrm{~kg}$, the optimization converges with satisfied constraints at a minimum energy per gait cycle of $E_{g c}=1.7 \mathrm{~J}$ and optimal parameters of $k_{s}=133 \mathrm{~N} \mathrm{~m} \mathrm{rad}^{-1}$ and $i_{G}=127$. Figure 3 presents the system behavior with the optimal stiffness and gear ratio. The dotted black line indicates $50 \%$ of the of the stride time. In the bottom115 right, the optimal series stiffness (green) differs from the quasi-stiffness of the knee. Comparing actuator power with knee power, the mechanical losses reduce possible recuperation in the swing phase and induce a positive power peak during stance. Due to little motion and high required torque in this phase and consequently low electric motor efficiency, a distinct increase in required electric power occurs. The required electrical peak power is $42.4 \mathrm{~W}$. The optimization

Table 1: Parameters and values for the exemplary drive system as given in the datasheets of the manufacturer

\begin{tabular}{cc}
\hline Parameters EC45 flat $(70 \mathrm{~W}, 24 \mathrm{~V})$ & Value \\
Rotor inertia $I_{m}$ & $1.81 \times 10^{-5} \mathrm{~kg} \mathrm{~m}^{2}$ \\
Torque Constant $k_{t}$ & $0.0369 \mathrm{~N} \mathrm{~m} \mathrm{~A}^{-1}$ \\
Speed Constant $k_{b}$ & $0.0369 \mathrm{~V} \mathrm{~s} \mathrm{rad}^{-1}$ \\
Terminal Resistance $R$ & $0.608 \Omega$ \\
Terminal inductance $L$ & $4.63 \times 10^{-4} \mathrm{H}$ \\
No Load Current $I_{n l}$ & $0.234 \mathrm{~A}$ \\
No Load Speed $\dot{\theta}_{n l}$ & $639.84 \mathrm{rad} \mathrm{s}^{-1}$ \\
maximum speed $\dot{\theta}_{m, \max }$ & $1047.2 \mathrm{rad} \mathrm{s}^{-1}$ \\
continuous current $I_{m, c o n}$ & $3.21 \mathrm{~A}$ \\
nominal voltage $U_{\max }$ & $24 \mathrm{~V}$ \\
\hline Parameters EPOS 24/5 & Value \\
efficiency $\eta_{m d}$ & 0.92 \\
maximum current $I_{m d, \max }$ & $10 \mathrm{~A}$ \\
\hline
\end{tabular}


result for a SEA is unconstrained due to preselection of a motor with sufficient continuous power.

In order to provide high efficiency throughout the whole gait cycle, the impact
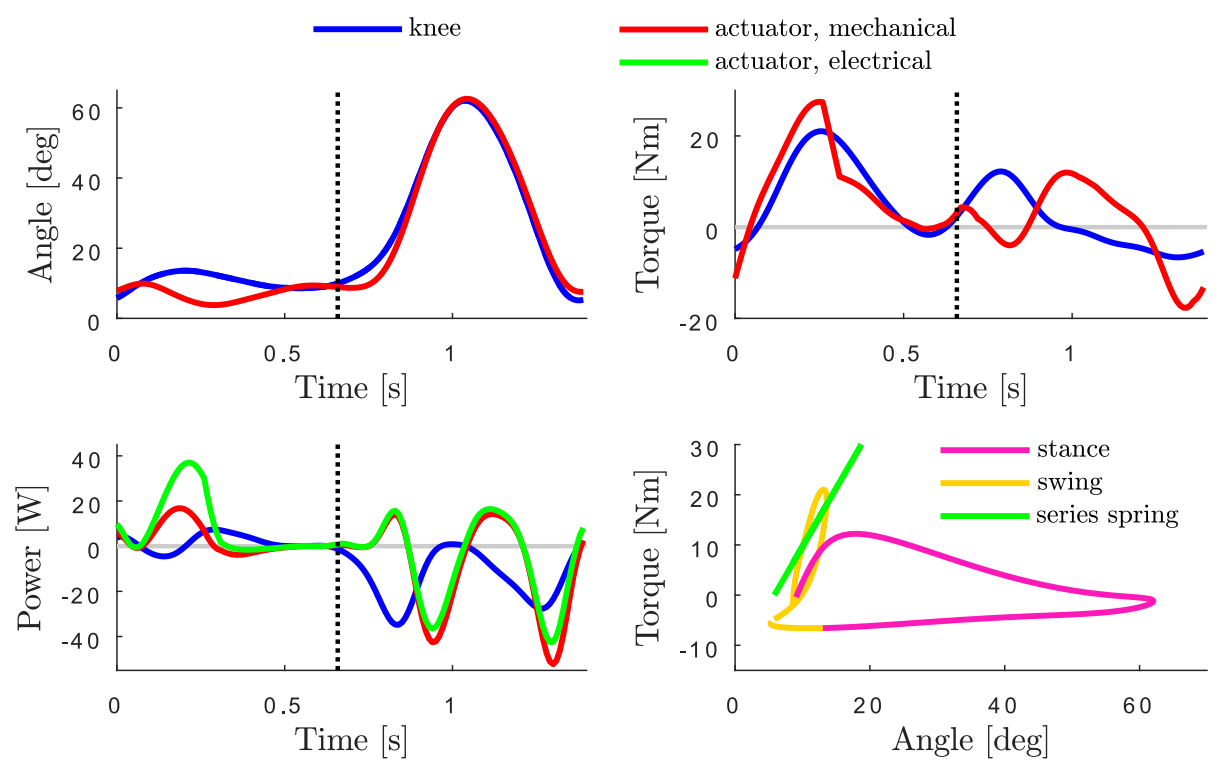

Figure 3: Behavior of a SEA with actuator inertia and total efficiency, $i_{G}=127$ and $k_{s}=$ $133 \mathrm{~N} \mathrm{~m} \mathrm{rad}^{-1}$, the dotted black line indicates $50 \%$ of the of the stride time. High torque in combination with little actuator motion during the stance phase requires high electrical peak power (bottom-left). The optimal series stiffness differs from the quasi-stiffness of the knee (bottom-right).

of locking the actuator position during stance phase is considered. In general,

this can be achieved by either utilizing a non-backdrivable gear unit or by adding a locking device [30]. The motion of the knee would then be based on deflection of the spring due to the knee load, storing energy in the spring, which is released to aid knee flexion in the beginning of the stance phase. As a non-backdrivable gear unit would prevent recuperation during swing phase, applying a lockingmechanism would be advantageous from an energetic point of view.

\subsection{Constrained Optimization of a Clutchable Series Elastic Actuator}

A CSEA for a prosthetic knee is proposed in [17, which employs an electromagnetic friction brake on the motor shaft as a clutch to achieve the required locking torque. A similar concept is realized by the weight acceptance mechanism in [18] and [31, utilizing a second electric motor with non-backdrivable spindle and spring, which is added in parallel to a SEA. In swing phase, the additional mechanism moves in a way, so that the SEA can move freely. The stiffness of the mechanisms in [17, 18, 31] is designed to passively mimic the torque-angle characteristic of the respective human joint. The concept of a 
Table 2: Parameters for the electromagnetic friction brake of the CSEA.

\begin{tabular}{cc}
\hline Parameters Combinorm 02.02.120 & Value \\
max. friction torque $\tau_{f t}$ & $0.75 \mathrm{~N} \mathrm{~m}$ \\
power consumption $P_{f t}$ & $6 \mathrm{~W}$ \\
Inertia $I_{f t}$ & $2.1 \times 10^{-6} \mathrm{~kg} \mathrm{~m}^{2}$ \\
\hline
\end{tabular}

CSEA is adopted and an actuator for an active knee orthosis is proposed for the comparison to the optimized SEA. Therefore, the electromagnetic friction brake (Combinorm 02.02.120, KEB, Barntrup, Germany), employed in [17, is implemented on the motor axis while considering the parameters given in Table 2 in the dynamic system models. When active, the brake is assumed to constantly generate a friction torque $\tau_{f t}$ of $0.75 \mathrm{Nm}$ while consuming $6 \mathrm{~W}$. To ensure that the locking by the brake is sufficient during stance phase, the constraint

$$
\tau_{k}<i_{G} \tau_{f t}
$$

is additionally implemented in the optimization. For the CSEA and the same gait parameters as used for the SEA $\left(t_{g c}=1.4 \mathrm{~s}, m_{h}=75 \mathrm{~kg}\right)$, the optimization converges at a minimum energy per gait cycle of $E_{g c}=-0.49 \mathrm{~J}$, thus allowing for recuperation of energy. The required electric peak power for the CESA is $-24.3 \mathrm{~W}$. The constraints are satisfied while the quasi-stiffness is determined as $k_{s}=295 \mathrm{~N} \mathrm{~m} \mathrm{rad}^{-1}$ and the optimal gear ratio is found to be $i_{G}=73$.

Including the locking mechanism slightly alters the resulting knee motion and the torque-angle characteristic as depicted in the top-left and bottom-right of Fig. 4. respectively. Similar to the SEA, the optimal parameters for the CSEA are not restricted by constraints.

However, the characteristics are similar to the natural gait cycle while the differences are deemed negligible. As there is no torque required from the actuator in 145 the stance phase, the mechanical power is also zero as depicted in the top-right and bottom-left. Activating the clutch results in a constant electrical power requirement of $6 \mathrm{~W}$ in the stance phase and the high peak power observed for the SEA is successfully avoided. 

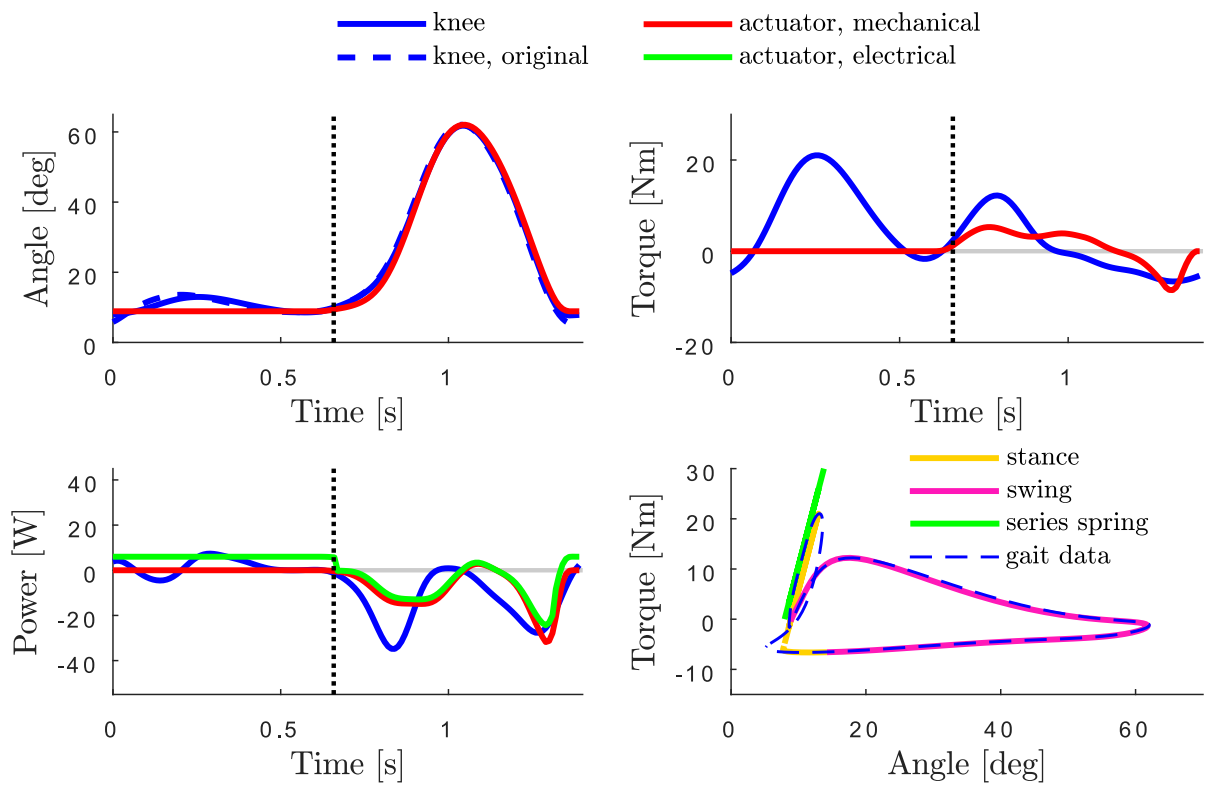

Figure 4: Behavior of a CSEA with actuator inertia and total efficiency, $i_{G}=73$ and $k_{s}=$ $295 \mathrm{~N} \mathrm{~m} \mathrm{rad}^{-1}$, the dotted black line indicates $50 \%$ of the of the stride time. The motor does not produce any torque during the first half of the gait cycle while the electromagnetic brake is active (top-right), resulting in constant electrical power consumption (bottom-left). The series stiffness mimics the quasi-stiffness of the knee (bottom-right).

\section{Impact of Friction and Gait Parameters} actual losses. In order to investigate these effects on the energy consumption of the SEA and the CSEA with optimal parameters found above, a parametric study is performed in the following.

\subsection{Method}

As gait characteristics may differ greatly across subjects and individual data is usually not available in literature, three parameters are used to scale the gait data given in [23]. The first parameter is the mass of the subject $m_{h}$ as the gait data is given in $\mathrm{N} \mathrm{m} \mathrm{kg}^{-1}$ and thus affects the knee load. The time per gait cycle $t_{g c}$ can be varied to investigate the impact of different walking velocities. Furthermore, a scaling factor for the knee angle $f_{k}$ is introduced to consider different knee flexion-extension rotations. In order to investigate the impact of the efficiency of gear units with high gear ratio, the constant efficiency factor $\eta_{g u}$ 
is replaced by a friction model containing Coulomb and viscous friction given by

$$
\tau_{f}=\mu_{v, a} \dot{\theta}_{a}+\mu_{c, a} \operatorname{sgn}\left(\dot{\theta}_{a}\right)
$$

with coefficients for Coulomb and viscous friction $\mu_{c, a}$ and $\mu_{v, a}$, respectively. Thus, instead of Eq. (4), the motor torque $\tau_{m}$ is determined by combining Eq. (3) and Eq. (13):

$$
\tau_{m}=I_{m} i_{G}\left(\frac{\ddot{\tau}_{k}}{k_{s}}+\ddot{\theta}_{k}\right)+\frac{\tau_{k}}{i_{G}}+\mu_{v, a} \dot{\theta}_{m}+\frac{\mu_{c, a}}{i_{G}} \operatorname{sgn}\left(\dot{\theta}_{m}\right) .
$$

The influence of varying motor friction is not investigated by the parametric study, the assumption for constant viscous damping $\nu_{m}$ in Eq. (6) is maintained.

The maximum value for the Coulomb and viscous friction coefficients are selected to cover the range of potential gear units and are in the magnitude of the 65 characteristic friction of a harmonic drive gear unit examined in [32, declared with respect to the output shaft of the gear unit. Mass of subjects $m_{h}$ is selected to approximately cover the adult body weight from 10 th to 90 th percentile. The minimum of $f_{k}$ reflects experience from a study performed with an active knee orthosis utilized by a subject with incomplete spinal cord injury 33. The limit for the time per gait cycle $t_{g c}=4 \mathrm{~s}$ is selected based on individual simulations with maximum friction coefficients in order to observe the impact of friction on constraints.

Minimum and maximum values as well as increments used in the parametric study are given in Table 3

Table 3: Overview of varied parameters in the parametric study

\begin{tabular}{cccc}
\hline Parameter & Minimum & Maximum & Increment \\
$m_{h}$ & $50 \mathrm{~kg}$ & $90 \mathrm{~kg}$ & $5 \mathrm{~kg}$ \\
$t_{g c}$ & $1 \mathrm{~s}$ & $4 \mathrm{~s}$ & $0.2 \mathrm{~s}$ \\
$f_{k}$ & 0.6 & 1.2 & 0.1 \\
$\mu_{c, a}$ & $0 \mathrm{~N} \mathrm{~m}$ & $10 \mathrm{~N} \mathrm{~m}$ & $0.25 \mathrm{~N} \mathrm{~m}$ \\
$\mu_{v, a}$ & $0 \mathrm{~N} \mathrm{~m} \mathrm{srad}^{-1}$ & $10 \mathrm{~N} \mathrm{~m} \mathrm{srad}^{-1}$ & $0.25 \mathrm{~N} \mathrm{~m} \mathrm{sad}^{-1}$ \\
\hline
\end{tabular}

\subsection{Results}

The parametric study yields a five-dimensional correlation matrix, describing the impact of all parameter combinations on the consumed energy per gait cycle $E_{g c}$. Exemplary results are discussed by means of Figure 5 , showing isolines for Coulomb friction from $0 \mathrm{Nm}$ to $10 \mathrm{Nm}$ in increments of $1 \mathrm{Nm}$. The corresponding viscous friction parameter is selected to be $\mu_{v, a}=0.5 \mu_{c, a}$ in

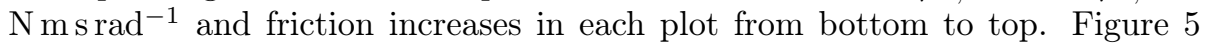
depicts the results for individual variation of each parameter, i.e., $m_{h}$ in the top 
plot, $t_{g c}$ in the middle, and $f_{k}$ in the bottom, while the non-varied parameters are taken from the original gait data $\left(t_{g c}=1.4 \mathrm{~s}, m_{h}=75 \mathrm{~kg}\right.$ and $\left.f_{k}=1\right)$.
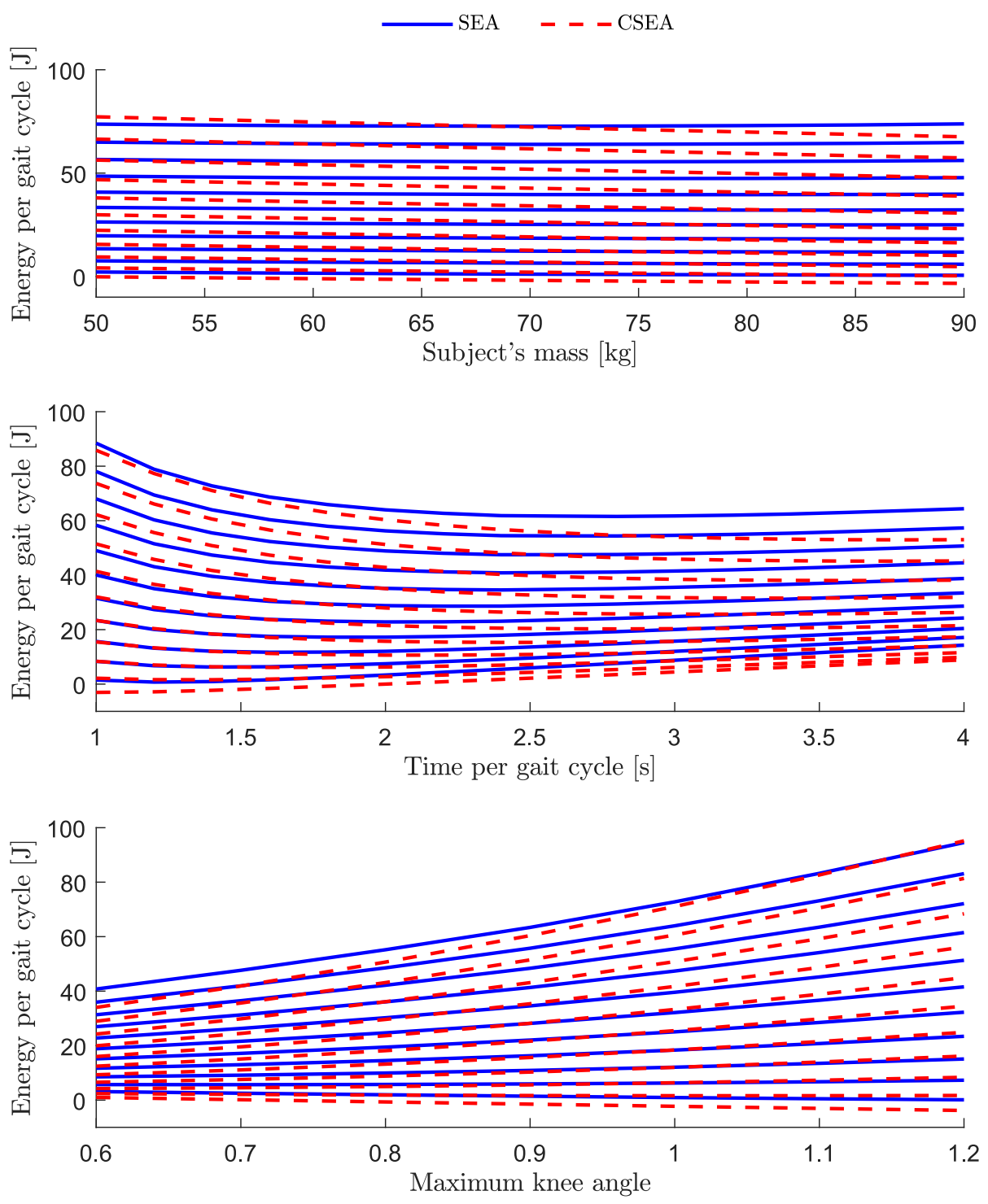

Figure 5: Result of the parametric study: comparison of the impact of subject's mass, time per gait cycle and knee angle for SEA and CSEA. Friction parameters increase from bottom to top in each plot.

185 The top of Fig. 5 shows little impact of $m_{h}$ on the energy consumption of the SEA compared to the impact of increasing friction. For the CSEA, an increasing $m_{h}$ yields higher knee torque, thus storing more energy in the spring during the 
stance phase, while the consumption of the locking mechanism is constant and load-independent. Thus, the consumed energy for the CSEA slightly decreases with increasing $m_{h}$. The increase of $E_{g c}$ due to friction only is similar for SEA and CSEA.

In contrast, variation of $t_{g c}$, which is negatively correlated to the knee velocity, demonstrates a different behavior with lower impact of friction for the CSEA than the SEA as depicted in the middle of Fig. 5. For both systems, the curves 195 for the required energy are convex, indicating a minimum depending on friction and cadence. Further, the results of the parametric study show reduced energy consumption for the CSEA, with increasing difference for slow ambulation.

The bottom plot of Fig. 5 shows increased energy consumption for increasing maximum amplitude of the knee motion, which is positive correlated to the knee velocity, for high friction. However, for friction parameters below $\mu_{c, a}=2 \mathrm{~N} \mathrm{~m}$ and $\mu_{v, a}=1 \mathrm{Nmsrad}^{-1}$, the required energy decreases when the maximum knee angle increases. This can be attributed to increased spring deflection during the stance phase and therefore the stored energy in the series spring increases as well. The general behavior for SEA and CSEA are similar, while the curves 205 are rising monotonously in the examined range of parameters in contrast to the variation of $t_{g c}$.

While the interrelation of the friction and gait parameters is difficult to assess, the parametric study indicates that $m_{h}$ has the least impact on required energy per gait cycle, while knowledge about actual gait and friction in the gear unit

${ }_{210}$ is required to reliably predict the energy consumption of an elastic actuation system.

Evaluating the impact of friction and gait parameters on actuator constraints provides the result presented in Fig. 6 for the SEA in the left and the CSEA in the right $\left(f_{k}=1\right)$. Each isoline with constant friction parameters indicates 215 the limit at which the constraints from Eq. (11) are still fulfilled on $t_{g c}$ and $m_{h}$. Thus, only motion at and above the isoline can be performed by the actuation system for the corresponding friction parameters. For both actuation principles, increasing friction limits the achievable cadence while the constraints for the SEA are found at a lower level of friction parameters. The SEA is lim-

220 ited by the nominal motor voltage $U_{m}$, which can be attributed to the selected gear ratio of $i_{G}=127$, which results from an optimization for $t_{g c}=1.4 \mathrm{~s}$. In addition, the dependency of constraints on the subject's mass $m_{h}$ shows that increasing knee load restricts the applicability of the SEA, as actuator torque and therefore motor current increase.

225 In contrast, high knee loads are advantageous for the CSEA, as actuator power and consumed energy are load-independent during the swing phase, as depicted in Fig. 4, and initially inspired to utilize a clutch. Higher loads thereby increase the energy stored in the spring and released during the swing phase, consequently reducing energy requirements. Furthermore, the CSEA is advantageous

230 for low friction, as constraints are active only for friction coefficients higher than $\mu_{c, a}=4 \mathrm{Nm}$ and $\mu_{v, a}=2 \mathrm{~N} \mathrm{~m} \mathrm{srad}-1$. The limiting constraint for the CSEA is the continuous motor current.

In contrast, for high friction coefficients, the achievable cadence and subject's 


$$
\begin{aligned}
&-\mu_{c, a}=2 \mathrm{Nm}, \mu_{v, a}=1 \mathrm{Nm} / \mathrm{s} \\
&-\mu_{c, a}=3 \mathrm{Nm}, \mu_{v, a}=1.5 \mathrm{Nm} / \mathrm{s} \\
& \mu_{c, a}=4 \mathrm{Nm}, \mu_{v, a}=2 \mathrm{Nm} / \mathrm{s} \\
&-\mu_{c, a}=7 \mathrm{Nm}, \mu_{v, a}=3.5 \mathrm{Nm} / \mathrm{s}
\end{aligned}
$$

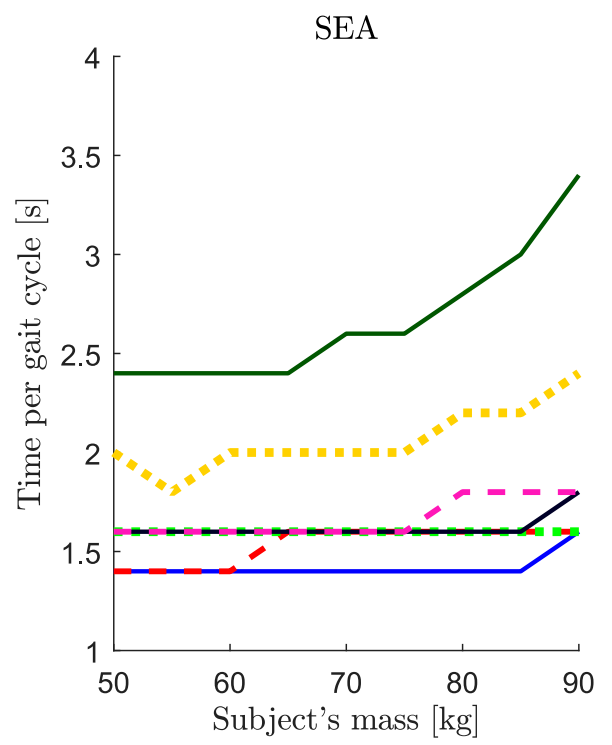

$$
\begin{aligned}
-\mu_{c, a} & =8 \mathrm{Nm}, \mu_{v, a}=4 \mathrm{Nm} / \mathrm{s} \\
\mu_{c, a} & =9 \mathrm{Nm}, \mu_{v, a}=4.5 \mathrm{Nm} / \mathrm{s} \\
\mu_{c, a} & =10 \mathrm{Nm}, \mu_{v, a}=5 \mathrm{Nm} / \mathrm{s}
\end{aligned}
$$

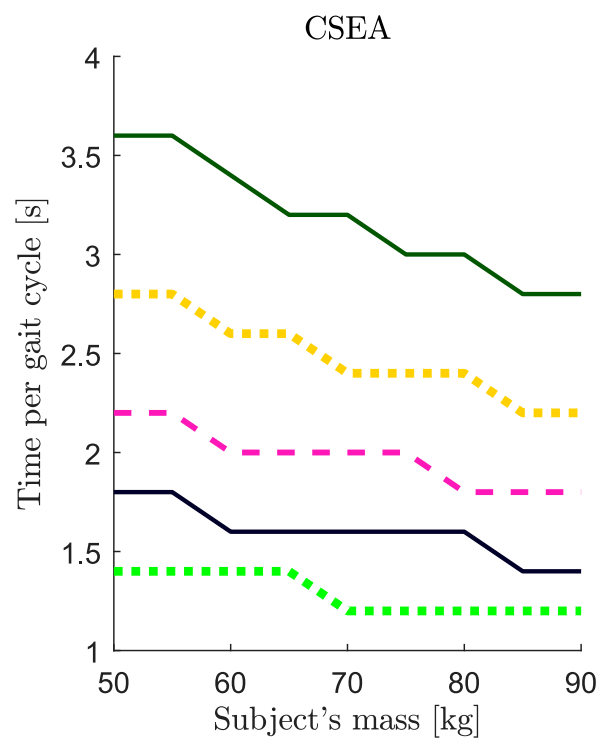

Figure 6: Result of the parametric study: Impact of gait parameters and selected friction parameters on actuator constraints of the SEA (left) and the CSEA (right). Only motions above the isoline with constant friction parameters for a given time per gait cycle and subjects mass can be performed by the actuation system. With the SEA, executable movements are limited for lower friction parameters than the CSEA. The SEA is less constrained for a low subject's mass, while the CSEA is favorable for higher subject's mass.

mass are higher for the SEA than for the CSEA. The transition in favor of the SEA occurs at approximately $\mu_{c, a}=7 \mathrm{Nm}$ and $\mu_{v, a}=3.5 \mathrm{Nms} \mathrm{rad}^{-1}$. For higher friction coefficients, active constraints in Fig. 6 for SEA and CSEA are concurrent in nominal voltage and continuous current, indicating that the continuous actuator power of $70 \mathrm{~W}$ is non-sufficient due to high losses in the gear unit. For lower friction parameters, only nominal voltage or continuous current impose limitations, showing the dependency of the optimized gear ratio to the selected gait data. Thus, optimizing the gear ratio for a range of gait velocities and knee loads may yield an actuation system with a wider applicability. However, a sound result requires knowledge about the friction parameters of the gear unit or a detailed efficiency model.

\section{Experimental Evaluation}

An experimental evaluation of the parametric study is performed via an energy analysis of a SEA and CSEA and comparison to the results of the models applied in Sections 2 and 3. Therefore, the direct drive system used in the 


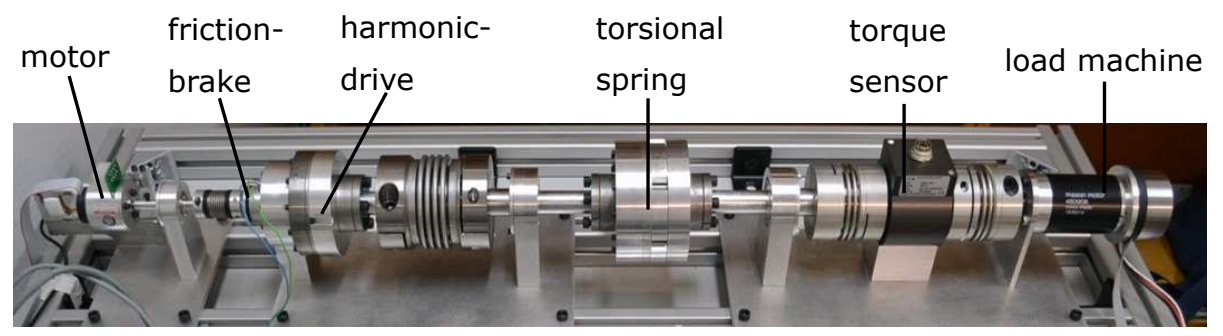

Figure 7: Test bench with load machine

low-cost robotic knee orthosis for individuals with spinal cord injury presented in [34 is extended by elastic components and a clutch (for the CSEA). This device includes the motor and motor controller considered in Section 2, A custom torsional spring was designed to introduce elastic behavior to the actuator. For the CSEA, an electromagnetic friction brake acts as the clutch. The utilized harmonic drive gear unit (SHD-20-160-2SH, Harmonic Drive AG, Limburg/Lahn,

255 Germany) [34, with a gear ratio of $i_{G}=160$, is retained despite the difference to the optimization results due to the low dimensions and high allowable torque.

\subsection{Experimental Setup}

A modular test bench was developed, allowing for a component-wise energy analysis of each component of the drive system. The load machine depicted

260 sured by a torque sensor DRBK-100 (ETH Messtechnik, Gschwend, Germany). Positions of actuator and knee are acquired by encoders at motor and load machine. A current sensor ACS712 (Allegro Microsystems, Worcester, Massachusetts, USA) is located after the power supply. Voltage is measured directly to determine the electric power consumption of the motor, motor driver and of the electromagnetic friction brake while the load machine is powered by a separate supply. Control algorithms and data recording is implemented on a MyRIO-1900 (National Instruments, Austin, Texas, USA). When not activating the clutch, the system behaves like a SEA, controlled by impedance control

270 adapted from [35]. For the CSEA, impedance control is deactivated while the electromagnetic friction brake is active during the stance phase. For implementation at the test bench, the gait phase is determined based on the temporal progression of the desired trajectories. The impedance control is extended by compensation of Coulomb and viscous friction with experimentally determined coefficients $\mu_{c, a}=6.8 \mathrm{Nm}$ and $\mu_{v, a}=2.6 \mathrm{~N} \mathrm{~ms} \mathrm{rad}^{-1}$. Furthermore, the reflected actuator inertia and the torsional stiffness were characterized prior to the experiments and yielded $I_{m}=3.4103 \times 10^{-5} \mathrm{~kg} \mathrm{~m}^{2}$ with respect to motor coordinates and $k=270 \mathrm{~N} \mathrm{~m} \mathrm{rad}{ }^{-1}$, respectively. In addition, an electric power consumption of approximately $6 \mathrm{~W}$ of the idle motor driver is observed and 280 considered as constant in the following. 


\subsection{Results}

Due to the high friction of the harmonic drive, a gait cycle with reduced motion is selected for the experimental evaluation of the models to reduce the required power. Figure 8 shows the results of the SEA performing a gait trajec-

285
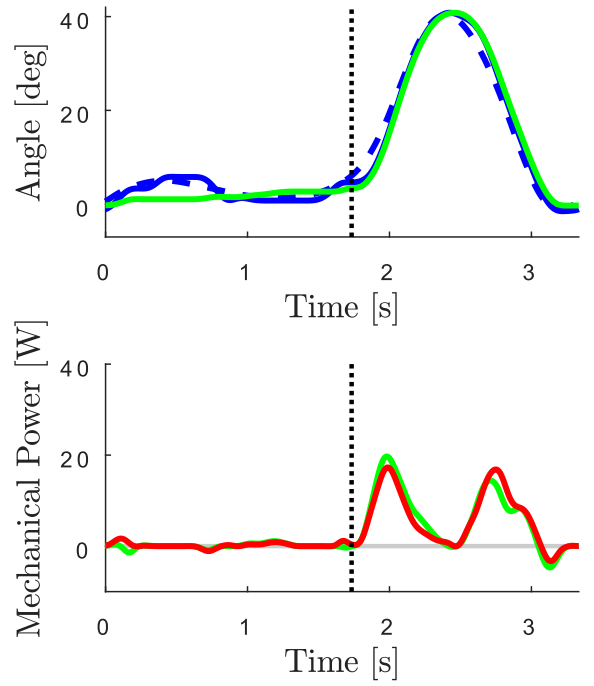
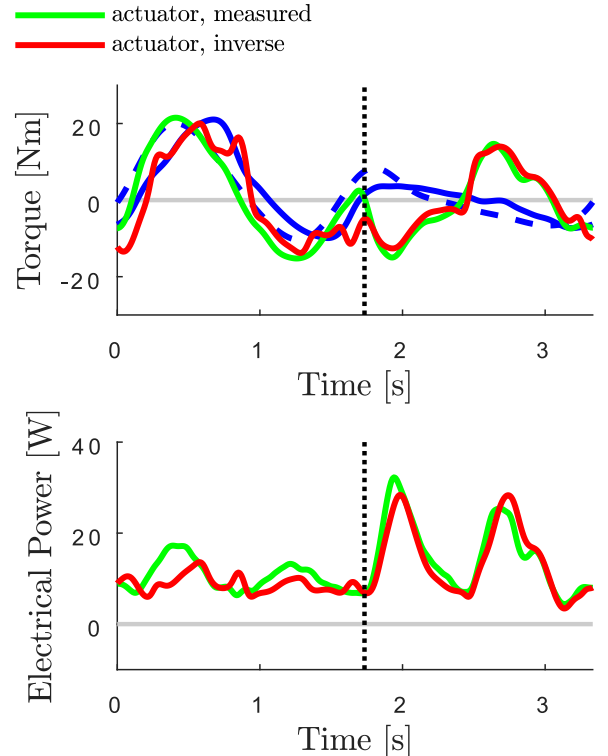

Figure 8: Performance of the SEA at the test bench during a gait cycle $\left(m_{h}=75 \mathrm{~kg}, f_{k}=0.66\right.$ and $\left.t_{g c}=3.33 \mathrm{~s}\right)$. The high friction of the gear unit reduces the required electrical peak power compared to Fig. 3 in the first half of the gait cycle, while distinctly increasing the required power in the second half (bottom-right).

The same motion is performed by the CSEA in Fig. 9, where the locked actuator position is directly visible as actuator motion, torque, and mechanical power are zero during the stance phase. The electrical power shows a superposition of 
the idle consumption of the motor driver and the constant consumption of the active electromagnetic brake during the stance phase. The achieved tracking performance as well as quality of models is similar for CSEA and SEA.
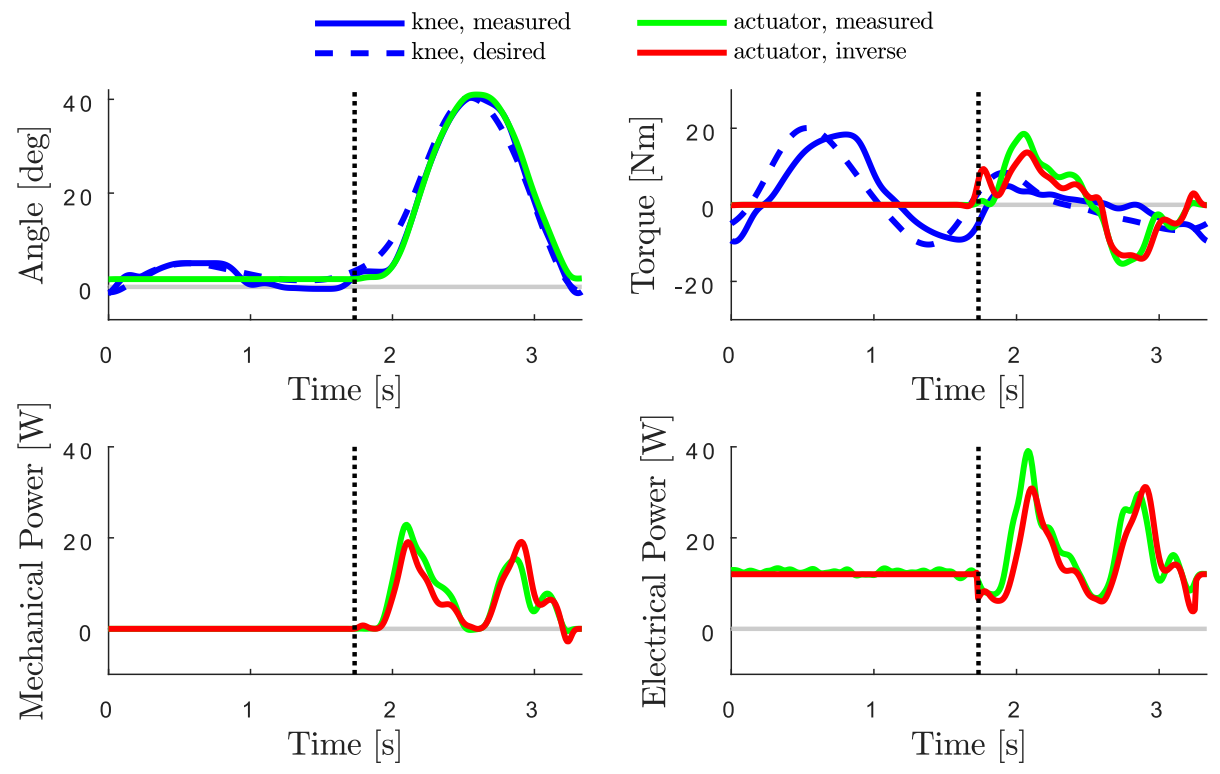

Figure 9: Performance of the CSEA at the test bench during a gait cycle $\left(m_{h}=75 \mathrm{~kg}, f_{k}=\right.$ 0.66 and $t_{g c}=3.33 \mathrm{~s}$ ). The electrical power consumption is constant while the electromagnetic brake is active and includes the idle consumption of the motor controller. Similar to the SEA, the high friction of the gear unit distinctly increases the required electrical power in the second half of the gait cycle.

The energy analysis of SEA and CSEA is presented in the left and right plot of Fig. 10 as a comparison of consumed power for the initial assumptions of component efficiency during the optimization, inverse calculations with the presented models and identified parameters at the test bench as well as measured values. Thus, losses are accumulated from the left to the right in each plot. As measurement of consumed electrical energy is not implemented component-wise, only the total consumed electrical energy is shown. Figure 10 shows a distinct underestimation of consumed energy with the initial assumptions for both SEA and CSEA. High accordance between inverse calculation and measured mechanical power indicates sufficient quality of the friction model to determine losses. The large increase between the consumed electrical energy of the motor and the motor driver originates from the constantly consumed approximately $6 \mathrm{~W}$ with high impact on the energy due to the large time per gait cycle $t_{g c}=3.33 \mathrm{~s}$. The total energy consumption of SEA and CSEA is $42.6 \mathrm{~J}$ and $48.2 \mathrm{~J}$, respectively, 320 and distinctly higher than with the initial assumptions. As the high friction of the gear unit limited the achievable dynamics of the executable gait cycle, the slow gait results in higher consumed energy per gait cycle for the CSEA than 
SEA

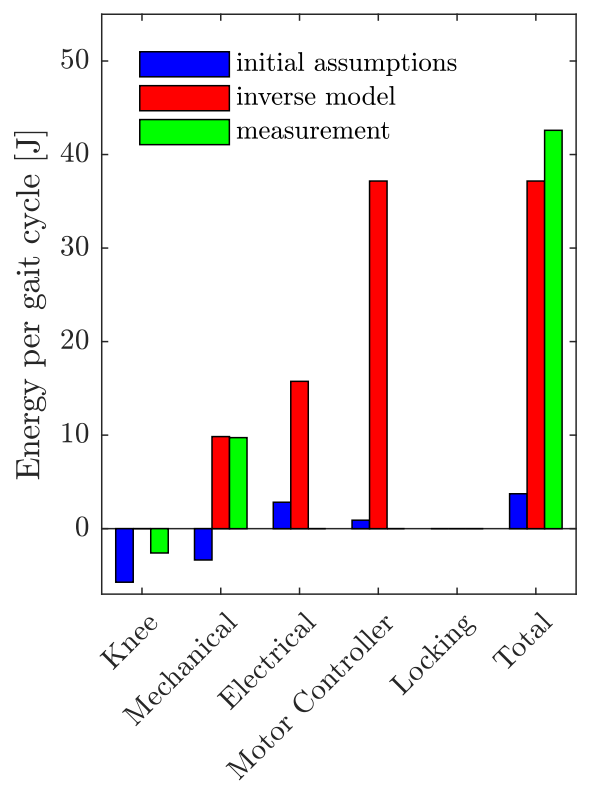

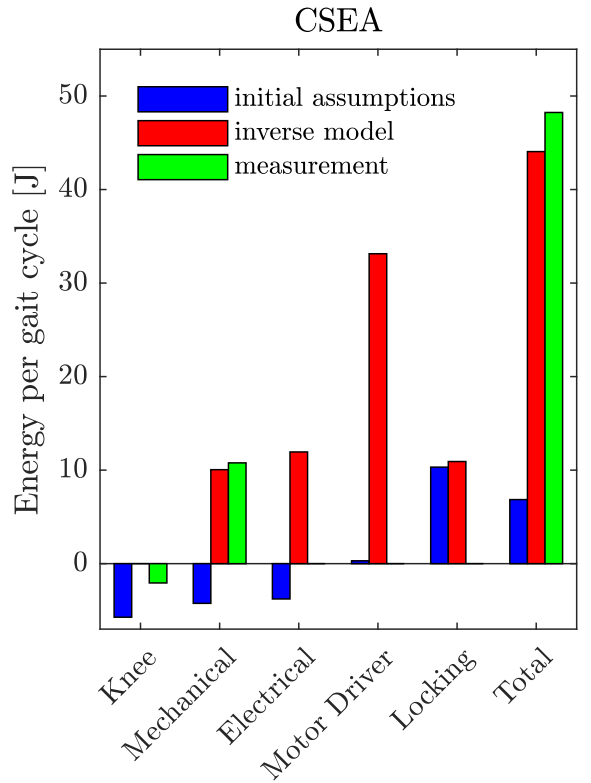

Figure 10: Comparison of consumed energy of each component for SEA (left) and CSEA (right). The improved calculation of the required energy per gait cycle with friction model compared to the constant efficiency made during the initial assumptions shows the importance of considering non-linear efficiency of components for the actuator design.

for the SEA due to constant power consumption of motor controller and electromagnetic brake. Comparing measurements and inverse modeled energy per gait cycle, the total consumption is underestimated by $5.4 \mathrm{~J}$ and $4.2 \mathrm{~J}$ for SEA and CSEA, respectively, indicating nonlinearities that are not considered by the models of both actuation systems. However, the predicted consumption of total electrical energy is distinctly improved by implementation of the friction model and idle consumption of the motor driver in contrast to the initial assumptions.

\section{Discussion}

Elastic actuators show great potential to reduce peak power, weight and energy consumption of wearable robots, while improving the physical human-robot interaction. To determine an optimal concept, configuration and parameters of the elastic actuation system, optimization techniques are applied, e.g., to minimize energy consumption. These optimizations often use healthy gait data as input for a model-based determination of the required torque, velocity and power. In order to evaluate the actuation concepts and parameters before the selection of individual components, general efficiency models improve the design procedure. This procedure was applied to a SEA and a CSEA to show the benefits of elastic actuation for given gait parameters in this paper. However, 
gait characteristics differ across subjects and situation, which can usually not be specified at the conceptual stage of actuator design.

As a compromise, especially regarding rehabilitation scenarios, we used the gait parameters time per gait cycle, maximum knee angle and subject's mass to scale gait data of healthy subjects for normal walking. The impact of varying parameters on energy consumption and actuator constraints of the optimized SEA and CSEA is analyzed via a parametric study. Furthermore, the constant efficiency of the gear unit is replaced with a Coulomb and viscous friction model to approximate non-linear losses without selecting a specific type of transmis-

350 sion. Variation of the friction parameters thereby yields insight into the relation of energy consumption and gait parameters and allows a more detailed comparison between SEA and CSEA. While increasing friction distinctly increases the consumed energy per step for both actuation concepts, especially the interrelationship to the time per gait cycle exhibits a nonlinear characteristic. Hence, characteristic maps of component efficiency or nonlinear loss models are required for realistic prediction of the energy consumption in assisted gait.

We demonstrated how friction in the gear unit limits the motions that can be performed by the actuation system by analysis of the actuator constraints, which depend on gait parameters and amount of friction. Especially the performance

360 of the SEA is limited for lower friction values than the CSEA. Comparing the dependency of constraints on the parameters shows advantages of the CSEA for high knee loads in contrast to the SEA. This can be attributed to the conceptual advantage of the CSEA and its load-independent power consumption of the electromagnetic brake in the stance phase.

365 To experimentally evaluate the computational models the study is based on, a modular test bench was utilized. Both SEA and CSEA were able to generate the desired motion and torque of a gait cycle on the test bench. However, the high experimentally evaluated friction in the transmission limited the examinable gait situations. To compare the SEA and the CSEA for a larger variety 370 of gait situations, the experiments should be repeated with an improved test bench. Comparison of mechanical and electrical power shows that the modelbased inverse calculation follows the experimental curves. The total consumed energy per gait cycle shows a distinct underestimation of losses compared to the initial assumptions with constant efficiencies for both SEA and CSEA. The

375 differences in mechanical losses can be attributed to the friction values of the utilized gear unit. Remaining deviations between inverse calculation and measurements could be reduced by considering further effects, e.g., friction of individual bearings at the test bench. It is important to highlight that the idle power consumption of the motor driver greatly impacts the total consumed 380 electric energy, which coincides with results presented in [25]. An evaluation of electrical power consumption of individual components was not possible with the test setup and should be performed to examine possible nonlinear efficiency of the motor driver, as the electric model of a brushless direct-current motor is well-examined. 


\section{Conclusion}

The results presented in this work highlight the interrelation of friction and gait parameters with energy consumption and actuator constraints, indicating that the state-of-the-art optimization based on healthy gait data does not necessarily result in an optimal actuator. In combination with the experimental evaluation, the results from the parametric study emphasize the necessity of detailed knowledge about nonlinear component efficiency as well as motion data to actually find an optimal actuator design. The parametric study points out that gait parameters distinctly influence the energy consumption of (clutchable) series elastic actuators. Moreover, the results imply that friction has less influence on the actuator constraints in clutchable actuators. Considering these effects improves the optimization and design of elastic actuators to achieve the desired reduction of peak power and energy consumption with elastic actuators.

\section{Acknowledgments}

400 The support of the Spanish Ministry of Economy and Competitiveness (MINECO) along with the European Regional Development Fund (ERDF) under project DPI2015-65959-C3-2-R is greatly acknowledged.

[1] N. A. Erosa, J. W. Berry, T. R. Elliott, A. T. Underhill, P. R. Fine, Predicting quality of life 5 years after medical discharge for traumatic spinal cord injury, British Journal of Health Psychology 19 (4) (2014) 688-700.

[2] World Health Organization, WHO global disability action plan 2014-2021, 2016.

[3] P. L. Ditunno, M. Patrick, M. Stineman, J. F. Ditunno, Who wants to walk? Preferences for recovery after SCI: a longitudinal and cross-sectional study, Spinal Cord 46 (7) (2008) 500-506.

[4] J. Veneman, E. Burdet, d. K. H. van, D. Lefeber, Emerging Directions in Lower Limb Externally Wearable Robots For Gait Rehabilitation and Augmentation - A Review, in: Advances in Cooperative Robotics, World Scientific Publishing Co. Pte. Ltd, 2017, pp. 840-850.

[5] M. Windrich, M. Grimmer, O. Christ, S. Rinderknecht, P. Beckerle, Active lower limb prosthetics: a systematic review of design issues and solutions, BioMedical Engineering OnLine 15 (S3).

[6] A. M. Dollar, H. Herr, Active Orthoses for the Lower-Limbs: Challenges and State of the Art, in: Rehabilitation Robotics, 2007. ICORR 2007. IEEE 10th International Conference on, 2007, pp. 968-977.

[7] H. Herr, Exoskeletons and orthoses: classification, design challenges and future directions, Journal of NeuroEngineering and Rehabilitation 6 (1) (2009) 21. 
[8] A. J. Young, D. P. Ferris, State of the Art and Future Directions for Lower Limb Robotic Exoskeletons, IEEE Transactions on Neural Systems and Rehabilitation Engineering 25 (2) (2017) 171-182.

[9] S. Au, H. Herr, Powered ankle-foot prosthesis, Robotics Automation Magazine, IEEE 15 (3) (2008) 52-59.

[10] M. Grimmer, M. Eslamy, S. Gliech, A. Seyfarth, A comparison of paralleland series elastic elements in an actuator for mimicking human ankle joint in walking and running, in: Robotics and Automation (ICRA), 2012 IEEE International Conference on, IEEE, 2012, pp. 2463-2470.

[11] B. Vanderborght, R. Van Ham, D. Lefeber, T. G. Sugar, K. W. Hollander, Comparison of mechanical design and energy consumption of adaptable, passive-compliant actuators, International Journal of Robotics Research 28 (1) (2009) 90-103.

[12] T. Verstraten, P. Beckerle, R. Furnémont, G. Mathijssen, B. Vanderborght, D. Lefeber, Series and Parallel Elastic Actuation: Impact of Natural Dynamics on Power and Energy Consumption, Mechanism and Machine Theory 102 (2016) 232-246.

[13] G. A. Pratt, M. M. Williamson, Series elastic actuators, in: IEEE/RSJ International Conference on Intelligent Robots and Systems, 1, Pittsburgh, 1995, pp. 399-406.

[14] G. Tonietti, R. Schiavi, A. Bicchi, Design and Control of a Variable Stiffness Actuator for Safe and Fast Physical Human/Robot Interaction., in: ICRA, IEEE, 2005, pp. 526-531.

[15] M. Grimmer, A. Seyfarth, Stiffness Adjustment of a Series Elastic Actuator in a Knee Prosthesis for Walking and Running: The Trade-off between Energy and Peak Power Optimization, in: IEEE/RSJ International Conference on Intelligent Robots and Systems, 2011.

[16] S. Wang, W. van Dijk, H. van der Kooij, Spring uses in exoskeleton actuation design, IEEE International Conference on Rehabilitation Robotics 2011 (2011) 5975471.

[17] E. J. Rouse, L. M. Mooney, H. M. Herr, Clutchable series-elastic actuator: Implications for prosthetic knee design, The International Journal of Robotics Research 33 (13) (2014) 1611-1625.

[18] L. Flynn, J. Geeroms, R. Jimenez Fabian, B. Vanderborght, D. Lefeber, CYBERLEGS Beta-Prosthesis active knee system, in: ICORR 2015 IEEE International Conference on Rehabilitation Robotics, 2015, pp. 410-415.

[19] D. A. Winter, Kinematic and kinetic patterns in human gait: Variability and compensating effects, Human Movement Science 3 (1-2) (1984) 51-76. 
[20] F. Danion, E. Varraine, M. Bonnard, J. Pailhous, Stride variability in human gait: the effect of stride frequency and stride length, Gait \& Posture 18 (1) (2003) 69-77.

[21] K. Jordan, J. H. Challis, K. M. Newell, Walking speed influences on gait cycle variability, Gait \& Posture 26 (1) (2007) 128-134.

[22] D. R. Louie, J. J. Eng, T. Lam, Gait speed using powered robotic exoskeletons after spinal cord injury: a systematic review and correlational study, Journal of NeuroEngineering and Rehabilitation 12 (2015) 82.

[23] G. Bovi, M. Rabuffetti, P. Mazzoleni, M. Ferrarin, A multiple-task gait analysis approach: kinematic, kinetic and EMG reference data for healthy young and adult subjects, Gait Posture 33 (1) (2011) 6-13.

[24] T. Verstraten, P. Beckerle, R. Furnémont, G. Mathijssen, B. Vanderborght, D. Lefeber, Series and Parallel Elastic Actuation: Impact of natural dynamics on power and energy consumption, Mechanism and Machine Theory 102 (2016) 232-246.

[25] J. Geeroms, L. Flynn, R. Jimenez-Fabian, B. Vanderborght, D. Lefeber, Energetic analysis and optimization of a MACCEPA actuator in an ankle prosthesis - energetic evaluation of the cyberlegs alpha-prosthesis variable stiffness actuator during a realistic load cycle, Auton. Robots 42 (1) (2018) $147-158$.

[26] T. Verstraten, J. Geeroms, G. Mathijssen, B. Convens, B. Vanderborght, D. Lefeber, Optimizing the power and energy consumption of powered prosthetic ankles with series and parallel elasticity, Mechanism and Machine Theory 116 (2017) 419-432.

[27] K. Shamaei, G. S. Sawicki, A. M. Dollar, Estimation of Quasi-Stiffness of the Human Knee in the Stance Phase of Walking, PLoS ONE 8 (3) (2013) $1-10$.

[28] T. Verstraten, G. Mathijssen, R. Furnémont, B. Vanderborght, D. Lefeber, Modeling and design of geared DC motors for energy efficiency: Comparison between theory and experiments, Mechatronics 30 (2015) 198-213.

[29] S. W. Lipfert, Kinematic and dynamic similarities between walking and running, Ph.D. thesis, Friedrich-Schiller Universität (2010).

[30] M. Plooij, G. Mathijssen, P. Cherelle, D. Lefeber, B. Vanderborght, Lock Your Robot: A Review of Locking Devices in Robotics, IEEE Robotics Automation Magazine 22 (1) (2015) 106-117.

[31] T. Bacek, M. Moltedo, K. Langlois, G. A. Prieto, M. C. SanchezVillamañan, J. Gonzalez-Vargas, B. Vanderborght, D. Lefeber, J. C. Moreno, BioMot exoskeleton \#x2014; Towards a smart wearable robot for symbiotic human-robot interaction, in: 2017 International Conference on Rehabilitation Robotics (ICORR), 2017, pp. 1666-1671. 
[32] T. D. Tuttle, Understanding and Modeling the Behavior of a Harmonic Drive Gear Transmission, Technical report, MIT, AI Lab. (May 1992).

[33] J. Font-Llagunes, A. Carnicero, J. Vidal, D. Clos, U. Lugrís, F. Alonso, J. Cuadrado, Able: assistive biorobotic low-cost exoskeleton, in: Simposio CEA de Bioingeniería, Asociación Nicolo, 2017, pp. 37-42.

[34] J. M. Font-Llagunes, D. Clos, U. Lugrís, F. Alonso, J., J. Cuadrado, Design and experimental evaluation of a low-cost robotic orthosis for gait assistance in subjects with spinal cord injury, in: Wearable Robotics: Challenges and Trends, Springer, 2017, pp. $281-285$.

[35] C. Ott, Cartesian Impedance Control of Redundant and Flexible-Joint Robots, Vol. 49 of Springer Tracts in Advanced Robotics, Springer Berlin Heidelberg, Berlin, Heidelberg, 2008. 